\title{
SOME NEW RESULTS ON CONFORMABLE FRACTIONAL POWER SERIES
}

\section{FRANCISCO MARTÍNEZ1,* , INMACULADA MARTÍNEZ , MOHAMMED K. A. KAABAR $^{2}$, SILVESTRE PAREDES ${ }^{1}$}

\author{
${ }^{1}$ Department of Applied Mathematics and Statistics, Technological University of Cartagena, \\ Spain \\ ${ }^{2}$ Department of Mathematics and Statistics, Washington State University, USA \\ *Corresponding author: f.martinez@upct.es \\ Received Jun. 14, 2020
}

Abstract. In this paper, some important results of the classical power series are generalized for the fractional power series. Some of these theorems are constructed by using conformable fractional derivatives. The ratio test has been specifically established to calculate the radius of convergence of a fractional power series, and several theorems of differentiability and integrability of the sum of a power series have been discussed in the sense of conformable fractional definition. In addition, the proposed series solution has been applied for the case of conformable fractional Airy differential equation.

2010 Mathematics Subject Classification. 26A33.

Key words and phrases. fractional power series; conformable fractional derivative; conformable fractional integral; conformable fractional differential equations.

\section{Introduction}

The idea of fractional derivative was raised first by L'Hospital in 1695. Since then, several related definitions have been proposed. The most common ones are RiemannLiouville and Caputo definitions. For more information about the most commonly known fractional definitions, we refer to [1,2]. Recently, a new definition of fractional 
derivative and fractional integral has been proposed by Khalil et al. in [3]. As a result, several important elements of the mathematical analysis of functions of a real variable have been proposed such as: Rolle's Theorem, Mean Value Theorem, chain rule, fractional power series expansion and fractional integration by parts formulas, [3-5]. The conformable partial derivative of the order $\alpha \in(0,1]$ of the real-valued functions of several variables and conformable gradient vector are defined, as well as a conformable version of Clairaut's Theorem for partial derivative is proven in [6]. In [7], conformable Jacobian matrix is defined and chain rule for multivariable conformable derivative is given. In [8], the conformable version of Euler's Theorem on homogeneous is introduced. Furthermore, in a short time, many studies have been conducted on the theory and applications of fractional differential equations based on this newly defined fractional derivative, [9-18].

This paper is organized as follows: In Section 2, the main concepts of conformable fractional calculus are presented. In Section 3, several important theorems are discussed; in particular, ratio test is established for a fractional power series, and the conformable differentiability and integrability of the sum of a power series is studied. In Section 4, the series solutions of conformable fractional Airy differential equation are obtained via the fractional power series technique.

\section{Basic Definitions and Tools}

Definition 2.1. Given a function $f:[0, \infty) \rightarrow R$. Then the conformable fractional derivative of $\operatorname{order} \alpha,[3]$, is defined by

$$
\left(T_{\alpha} f\right)(t)=\lim _{\epsilon \rightarrow 0} \frac{f\left(t+\varepsilon t^{1-\alpha}\right)-f(t)}{\varepsilon}
$$

for all $t>0,0<\alpha \leq 1$. If $f$ is $\alpha$-differentiable in some $(0, a), a>0$, and $\lim _{t \rightarrow 0^{+}}\left(T_{\alpha} f\right)(t)$ exist, then it is defined as 


$$
\left(T_{\alpha} f\right)(0)=\lim _{t \rightarrow 0^{+}}\left(T_{\alpha} f\right)(t)
$$

Theorem 2.1. [3]. If a function $f:[0, \infty) \rightarrow R$ is $\alpha$-differentiable at $t_{0}>0,0<\alpha \leq 1$, then $f$ is continuous at $t_{0}$.

Theorem 2.2. [3]. Let $0<\alpha \leq 1$ and $f, g$ be $\alpha$-differentiable at a point $t>0$. Then

(i) $\quad T_{\alpha}(a f+b g)=a\left(T_{\alpha} f\right)+b\left(T_{\alpha} g\right), \forall a, b \in R$.

(ii) $T_{\alpha}\left(t^{p}\right)=p t^{p-\alpha}, \forall p \in R$.

(iii) $\quad T_{\alpha}(\lambda)=0$, for all constant functions $f(t)=\lambda$.

(iv) $T_{\alpha}(f g)=f\left(T_{\alpha} g\right)+g\left(T_{\alpha} f\right)$.

(v) $T_{\alpha}\left(\frac{f}{g}\right)=\frac{g\left(T_{\alpha} f\right)-f\left(T_{\alpha} g\right)}{g^{2}}$.

(vi) If, in addition, $f$ is differentiable, then $\left(T_{\alpha} f\right)(t)=t^{1-\alpha} \frac{d f}{d t}(t)$.

The conformable fractional derivative of certain functions for the above definition is given as:

(i) $\quad T_{\alpha}(1)=0$,

(ii) $T_{\alpha}(\sin (a t))=a t^{1-\alpha} \cos (a t)$,

(iii) $T_{\alpha}(\cos (a t))-a t^{1-\alpha} \sin (a t)$,

(iv) $T_{\alpha}\left(e^{a t}\right)=a e^{a t}, a \in R$.

Definition 2.2. The (left) conformable derivative starting from a of a function $f:[a, \infty) \rightarrow R$ of $f$ of order $0<\alpha \leq 1,[4]$, is defined by

$$
\left(T_{\alpha}^{a} f\right)(t)=\lim _{\epsilon \rightarrow 0} \frac{f\left(t+\varepsilon(t-a)^{1-\alpha}\right)-f(t)}{\varepsilon}
$$

When $a=0$, it is written as $\left(T_{\alpha} f\right)(t)$. If $f$ is $\alpha$-differentiable in some $(a, b)$, then let us define the following:

$$
\left(T_{\alpha}^{a} f\right)(a)=\lim _{t \rightarrow a^{+}}\left(T_{\alpha}^{a} f\right)(t)
$$

Theorem 2.3 (Chain Rule). [4]. Assume $f, g:(a, \infty) \rightarrow R$ be (left) $\alpha$-differentiable functions, where $0<\alpha \leq 1$. Let $h(t)=f(g(t))$. The $h(t)$ is $\alpha$-differentiable for all $t \neq a$ and $g(t) \neq 0$, therefore, we have: 


$$
\left(T_{\alpha}^{a} h\right)(t)=\left(T_{\alpha}^{a} f\right)(g(t)) \cdot\left(T_{\alpha}^{a} g\right)(t) \cdot(g(t))^{\alpha-1}
$$

If $t=a$, then

$$
\left(T_{\alpha}^{a} h\right)(a)=\lim _{t \rightarrow a^{+}}\left(T_{\alpha}^{a} f\right)(g(t)) \cdot\left(T_{\alpha}^{a} g\right)(t) \cdot(g(t))^{\alpha-1}
$$

Theorem 2.4. [4]. Assume $f$ is infinitely $\alpha$-differentiable function, for some $0<\alpha \leq 1$ at a neighborhood of a point $t_{0}$. Then $f$ has the following fractional power series expansion:

$$
f(t)=\sum_{k=0}^{\infty} \frac{\left({ }^{(k)} T_{\alpha}^{t_{0}}\right)\left(t_{0}\right)}{\alpha^{k} k !}\left(t-t_{0}\right)^{k \alpha}, t_{0}<t<t_{0}+R^{\frac{1}{\alpha}}
$$

Here, $\left({ }^{(k)} T_{\alpha}^{t_{0}}\right)\left(t_{0}\right)$ means the application of the fractional derivative $k$ times.

The following definition for the $\alpha$-fractional integral of a function $f$ starting from $a \geq 0$.

Definition 2.3. $I_{\alpha}^{a}(f)(t)=\int_{a}^{t} \frac{f(x)}{x^{1-\alpha}} \cdot d x$, where the integral is the usual Riemann improper integral, and $\alpha \in(0,1],[3]$.

The most general sequential linear homogeneous conformable fractional differential equation is

$$
{ }^{(n)} T_{\alpha}^{a} y+a_{n-1}(t)^{(n-1)} T_{\alpha}^{a} y+\cdots+a_{1}(t) T_{\alpha}^{a} y+a_{0}(t) y=0
$$

where ${ }^{(n)} T_{\alpha}^{a} y=T_{\alpha}^{a} T_{\alpha}^{a} \ldots T_{\alpha}^{a} y, n$ times.

Definition 2.4. [9]. Let $\alpha \in(0,1], t_{0} \in[a, b] \subset[0, \infty), N\left(t_{0}\right)$ be a neighborhood of $t_{0}$ and $f(t)$ be a real function defined on $[a, b]$. In this case $f(t)$ is said to be $\alpha$-analytic at $t_{0}$ if $f(t)$ can be expressed as a series of natural powers of $\left(t-t_{0}\right)^{\alpha}$ for all $t \in N\left(t_{0}\right)$. In other words, $f(t)$ can be expressed as follows:

$$
\sum_{k=0}^{\infty} c_{k}\left(t-t_{0}\right)^{k \alpha}, \quad c_{k} \in R
$$

This series is definitely convergent for $t_{0}<t<t_{0}+\delta(\delta>0)$ where $\delta$ is the radius of convergence of the series.

Definition 2.5. [9]. Let $\alpha \in(0,1], t_{0} \in[a, b] \subset[0, \infty)$, and the functions $a_{k}(t)$ be $\alpha$-analytic at $t_{0} \in[a, b] \subset[0, \infty)$ for $k=0,1,2, \ldots, n-1$. In this case, the point $t_{0}$ is said to be an $\alpha$-ordinary point of (2.8). 
Theorem 2.5. [9]. Let $\alpha \in(0,1]$, let $c_{0}, c_{1} \in R$ and let $t_{0}$ be an $\alpha$-ordinary point of the equation

$$
T_{\alpha}^{t_{0}} T_{\alpha}^{t_{0}} y+p(t) T_{\alpha}^{t_{0}} y+q(t) y=0
$$

Then, there exists a solution to the Eq. (2.9) as:

$$
y=\sum_{k=0}^{\infty} c_{k}\left(t-t_{0}\right)^{k \alpha}
$$

for $t \in\left(t_{0}, t_{0}+\rho\right)$ with $\rho=\min \left\langle\delta_{1}, \delta_{2}\right\rangle$ and initial conditions: $c_{0}=y\left(t_{0}\right), \alpha c_{1}=T_{\alpha} y\left(t_{0}\right)$ where $\delta_{1}$ and $\delta_{2}$ are the radii of convergence of $p(t)$ and $q(t)$, respectively.

\section{Conformable Power Series Representation}

In this section, we will generalize some important related definitions and theorems from the classical power series into the fractional case in the conformable sense. New results related to the convergent of the series $\sum_{k=0}^{\infty} c_{k} t^{k \alpha}$ are also presented. After that, some results which are particularly focused on the radii of convergence for the conformable power series are utilized.

Definition 3.1. A power series representation of the form

$$
\sum_{k=0}^{\infty} c_{k}\left(t-t_{0}\right)^{k \alpha}=c_{0}+c_{1}\left(t-t_{0}\right)^{\alpha}+c_{2}\left(t-t_{0}\right)^{2 \alpha}+\ldots
$$

where $0<\alpha \leq 1$ and $t \geq t_{0} \geq 0$ is called conformable power series about $t_{0}$, and $c_{k}$ 's are constants known as the coefficients of the series.

As a special case, when $t_{0}=0$, the expansion $\sum_{k=0}^{\infty} c_{k} t^{k \alpha}$ is called a conformable Maclaurin series. Notice that in writing out the term corresponding to $k=0$ in equation (3.1), we have adopted the convention $\left(t-t_{0}\right)^{0}=1$ that even when $t=t_{0}$. Also, when $t=t_{0}$, each term of Eq. (3.1) vanishes for $k \geq 1$ and so. On the other hand, the conformable power series (3.1) is always convergent when $t=t_{0}$. For simplicity, we will only present the case when $t_{0}=0$ as follows:

Theorem 3.1. We have the following two cases for the conformable power series $\sum_{k=0}^{\infty} c_{k} t^{k \alpha}$, $t \geq 0$ : 
(i) If the conformable power series $\sum_{k=0}^{\infty} c_{k} t^{k \alpha}$ converges when $t=b>0$, then it converges whenever $0 \leq t<b$.

(ii) If the conformable power series $\sum_{k=0}^{\infty} c_{k} t^{k \alpha}$ diverges when $t=d>0$, then it converges whenever $t>d$.

Proof. For the first part, suppose that $\sum c_{k} b^{k \alpha}$ converges. Then, we have: $\lim _{k \rightarrow \infty} c_{k} b^{k \alpha}=0$. According to the definition of the limit of sequences with $\varepsilon=1$, there is a positive integer $N$ such that $\left|c_{k} b^{k \alpha}\right|<1$ whenever $k \geq N$. Thus, for $k \geq N$, we have: $\left|c_{k} t^{k \alpha}\right|=$ $\left|\frac{c_{k} b^{k \alpha} t^{k \alpha}}{b^{k \alpha}}\right|=\left|c_{k} b^{k \alpha}\right|\left|\frac{t}{b}\right|^{k \alpha}<\left|\frac{t}{b}\right|^{k \alpha}$. Again, if we have: $0 \leq t<b$, then $\left|\frac{t}{b}\right|^{\alpha}<1$, so $\sum\left|\frac{t}{b}\right|^{k \alpha}$ is a convergent geometric series. Therefore, by the comparison test, the series $\sum_{k=N}^{\infty}\left|c_{k} t^{k \alpha}\right|$ is convergent. Thus, the series $\sum c_{k} t^{k \alpha}$ is absolutely convergent, and therefore, it is convergent. To prove the remaining part, suppose that diverges. Now, if there is any number such that $t>d>0$, then $\sum c_{k} t^{k \alpha}$ cannot converge because, by case 1 , the convergence of $\sum c_{k} t^{k \alpha}$ would imply the convergence of $\sum c_{k} d^{k \alpha}$. Therefore, $\sum c_{k} t^{k \alpha}$ diverges whenever $t>d$. This completes the proof.

Theorem 3.2. For the conformable power series $\sum_{k=0}^{\infty} c_{k} t^{k \alpha}, t \geq 0$, there are only three possibilities:

(i) The series $\sum c_{k} t^{k \alpha}$ converges only when $t=0$,

(ii) The series $\sum c_{k} t^{k \alpha}$ converges for each $t \geq 0$,

(iii) There is a positive real number $R$ such that the series converges whenever $0 \leq t<R$ and diverges whenever $t>R$.

Proof. Suppose that neither case 1 nor case 2 is true. Then, there are non-zero numbers $b$ and $\mathrm{d}$ such that $\sum c_{k} t^{k \alpha}$ converges for $t=b$ and diverges for $t=d$. Therefore, the set $S=$ $\left\{t: \sum c_{k} t^{k \alpha}\right.$ converges $\}$ is not empty. By the preceding theorem, the series diverges if $t>$ $d$, so $0 \leq t<d$ for each $t \in S$. This says that $d$ is an upper bound for $S$. Thus, by the completeness axiom, it has a least upper bound $R$. If $t>R$, then $t \notin S$, so $\sum c_{k} t^{k \alpha}$ diverges. If $0 \leq t<b$, then $t$ is not an upper bound for $S$ and so there exists $b \in S$ such 
that $b>t$. Since $b \in S$ and $\sum c_{k} t^{k \alpha}$ converges, so by the preceding theorem, $\sum c_{k} t^{k \alpha}$ converges, so the proof of the theorem is complete.

Remark 3.1. The number $R$ in case 3 of theorem 3.2 is called the radius of convergence of the conformable power series. By convention, the radius of convergence is $R=0$ in case 1 and $R=\infty$ in case 2 .

Remark 3.2. Suppose that the following conformable power series $\sum_{k=0}^{\infty} c_{k} t^{k \alpha}, t \geq 0$ has the radius of convergence $R \geq 0$. As in the classical case, [19], it is easy to prove that if $0<\rho<R$, then the conformable power series converges uniformly on the interval $[0, \rho]$, and the sum of the series is continuous in $[0, R]$.

Remark 3.3. To study the convergence of the conformable power series, we test that by ratio test as follows, [10]:

$$
\lim _{k \rightarrow \infty}\left|\frac{c_{k} t^{k \alpha}}{c_{k+1} t^{(k+1) \alpha}}\right|=\lim _{k \rightarrow \infty}\left|\frac{c_{k}}{c_{k+1} t^{\alpha}}\right|
$$

Now if we assume $l=\lim _{k \rightarrow \infty}\left|\frac{c_{k}}{c_{k+1}}\right|$, then:

(i) If $l=0$, the series will diverge for all $t \neq 0$.

(ii) If $0<l<\infty$, then $\sum c_{k} t^{k \alpha}$ converges if $0 \leq t<l^{\frac{1}{\alpha}}$ and diverges if $t>l^{\frac{1}{\alpha}}$.

(iii) If $l=\infty$, the series converges for all $t \in[0, \infty)$.

\section{Example 3.1.}

(i) The geometric series $\sum_{k=0}^{\infty} t^{k \alpha}=1+t^{\alpha}+t^{2 \alpha}+\cdots$ has radius of convergence $R=1$, since $l=\lim _{k \rightarrow \infty}\left|\frac{c_{k}}{c_{k+1}}\right|=1$. At $t=1$, the series becomes $1+1+1+1+\cdots$, which diverges. Thus, the interval of convergence of the power series is $[0,1)$. The series converges uniformly on $\lceil 0, \rho\rceil$ for every $0<\rho<1$, but it does not converge uniformly on $[0,1)$.

(ii) Let $0<\alpha<1$. The power series $\sum_{k=0}^{\infty} \frac{t^{k \alpha}}{\alpha^{k} k !}=1+\frac{t^{\alpha}}{\alpha}+\frac{t^{2 \alpha}}{\alpha^{2} 2 !}+\frac{t^{3 \alpha}}{\alpha^{3} 3 !}+\cdots$ has a radius of convergence $R=\infty$, since $l=\lim _{k \rightarrow \infty}\left|\frac{c_{k}}{c_{k+1}}\right|=\lim _{k \rightarrow \infty}\left|\frac{\alpha^{k+1}(k+1) !}{\alpha^{k} k !}\right|=$ $\lim _{k \rightarrow \infty} \alpha(k+1)=\infty$ 
(iii) The power series $\sum_{k=0}^{\infty}(k !) t^{k \alpha}=1+t^{\alpha}+(2 !) t^{2 \alpha}+(3 !) t^{3 \alpha}+\cdots$ has a radius of convergence $R=0$, since $l=\lim _{k \rightarrow \infty}\left|\frac{c_{k}}{c_{k+1}}\right|=\lim _{k \rightarrow \infty}\left|\frac{k !}{(k+1) !}\right|=\lim _{k \rightarrow \infty} \frac{1}{k+1}=0$.

Remark 3.4. As in the classical case, we will show that the sum of a conformable power series $f(t)=c_{0}+c_{1} t^{\alpha}+c_{2} t^{2 \alpha}+c_{3} t^{3 \alpha}+\cdots$ is infinitely $\alpha$-differentiable inside its interval of convergence, and its $\alpha$-derivative $\left(T_{\alpha} f\right)(t)=\alpha c_{1}+2 \alpha c_{2} t^{\alpha}+3 \alpha c_{3} t^{2 \alpha}+\cdots$ is given by the term-by-term differentiation. To prove this, we first show that the term-byterm $\alpha$-derivative of a power series has the same radius of convergence as the original power series.

Theorem 3.3. Suppose that the power series $\sum_{k=0}^{\infty} c_{k} t^{k \alpha}$ has radius of convergence $R$. Then the power series $\sum_{k=1}^{\infty} k \alpha c_{k} t^{(k-1) \alpha}$ also has radius of convergence $R$.

Proof. Suppose $0 \leq t<R$. Choose $\rho$ such that $0 \leq t<\rho<R$, and let $r=\frac{t}{\rho}, 0<r<1$.

To estimate the terms in the $\alpha$-differentiated power series by the terms in the original series, we re-write their absolute values as follows:

$$
\left|k \alpha c_{k} t^{(k-1) \alpha}\right|=\frac{k \alpha}{\rho^{\alpha}}\left(\frac{t}{\rho}\right)^{(k-1) \alpha}\left|c_{k} \rho^{k \alpha}\right|=\frac{k \alpha r^{(k-1) \alpha}}{\rho^{\alpha}}\left|c_{k} \rho^{k \alpha}\right|
$$

The ratio test shows that $\sum k \alpha c_{k} t^{(k-1) \alpha}$ converges since $\lim _{k \rightarrow \infty}\left|\frac{k r^{(k-1) \alpha}}{(k+1) r^{k \alpha}}\right|=\lim _{k \rightarrow \infty}[(1-$ $\left.\left.\frac{1}{k+1}\right) \frac{1}{r^{\alpha}}\right]=\frac{1}{r^{\alpha}}>1$.

Hence, the sequence $k r^{(k-1) \alpha}$ is bounded, say by $M$. It follows that $\left|k \alpha c_{k} t^{(k-1) \alpha}\right| \leq$ $\frac{M}{\rho^{\alpha}}\left|c_{k} \rho^{k \alpha}\right|$ for all $k \in N$. The series $\sum\left|c_{k} \rho^{k \alpha}\right|$ converges, since $\rho<R$, so the comparison test implies that $\sum k \alpha c_{k} t^{(k-1) \alpha}$ converges absolutely. On the contrary, suppose that $t>$ $R$. Then, $\sum\left|c_{k} t^{k \alpha}\right|$ diverges (since $\sum c_{k} t^{k \alpha}$ diverges) and $\left|k \alpha c_{k} t^{(k-1) \alpha}\right| \geq \frac{\alpha}{t}\left|c_{k} t^{k \alpha}\right|$ for $k \geq$ 1 , so the comparison test implies that $\sum k \alpha c_{k} t^{(k-1) \alpha}$ diverges. Thus, the series have the same radius of convergence.

Theorem 3.4. Suppose that the power series $f(t)=\sum_{k=0}^{\infty} c_{k} t^{k \alpha}$ for all $t \in[0, R)$, has the radius of convergence $R>0$ and sum $f$. Then, $f$ is $\alpha$-differentiable in $[0, R)$, and 
$\left(T_{\alpha} f\right)(t)=\sum_{k=1}^{\infty} k \alpha c_{k} t^{(k-1) \alpha}$.

Proof. The term-by-term differentiated conformable power series converges in $[0, R)$ by theorem 3.3. Denote its sum by $g(t)=\sum_{k=1}^{\infty} k \alpha c_{k} t^{(k-1) \alpha}$. Let $0<\rho<R$. Then, by remark 3.2, the power series for $f$ and $g$ both converge uniformly in $[0, \rho)$. Applying the classical result on the differentiability of the sequences of functions, [19], to their succession of partial sums, we conclude that $f$ is differentiable in $[0, \rho)$ and $\left(T_{\alpha} f\right)(t)=$ $g(t)$. Since this holds for every $0<\rho<R$, it follows that $f$ is differentiable in $[0, R)$ and $\left(T_{\alpha} f\right)(t)=g(t)$, which proves the result. Repeated application of theorem 3.4 implies that the sum of a power series is infinitely $\alpha$-differentiable inside its interval of convergence and its derivatives are given by the term-by-term differentiation of the conformable power series. Furthermore, we can get an expression for the coefficients $c_{k}$ in terms of the function $f$; they are simply known as the conformable Taylor coefficients of $f$ at 0 .

Theorem 3.5. If the power series $f(t)=\sum_{k=0}^{\infty} c_{k} t^{k \alpha}$ has a radius of convergence $R>0$, then $f$ is infinitely $\alpha$-differentiable in $[0, R)$, and $c_{k}=\frac{\left({ }^{(k)} T_{\alpha}\right)(0)}{\alpha^{k} k !}$.

Proof. By applying theorem 3.4 to the power series $f(t)=c_{0}+c_{1} t^{\alpha}+c_{2} t^{2 \alpha}+c_{3} t^{3 \alpha}+$ $\cdots+c_{k} t^{k \alpha}+\ldots, n$ times, we find that $f$ has $\alpha$-derivatives of every order in $[0, R)$, and

$$
\begin{gathered}
\left({ }^{(1)} T_{\alpha} f\right)(t)=\alpha c_{1}+2 \alpha c_{2} t^{\alpha}+3 \alpha c_{3} t^{2 \alpha}+\cdots+k \alpha c_{k} t^{(k-1) \alpha}+\cdots \\
\left({ }^{(2)} T_{\alpha} f\right)(t)=2 \alpha^{2} c_{2}+(3 \cdot 2) \alpha^{2} c_{3} t^{2 \alpha}+\cdots+k(k-1) \alpha^{2} c_{k} t^{(k-1) \alpha}+\cdots \\
\left({ }^{(3)} T_{\alpha} f\right)(t)=(3 \cdot 2 \cdot 1) \alpha^{3} c_{3} t^{\alpha}+\cdots+k(k-1)(k-2) \alpha^{3} c_{k} t^{(k-2) \alpha}+\cdots \\
\vdots \\
\left({ }^{(n)} T_{\alpha} f\right)(t)=(n !) \alpha^{n} c_{n}+\cdots+\frac{k !}{(k-n) !} \alpha^{n} c_{k} t^{(k-n) \alpha}+\cdots
\end{gathered}
$$

where all of these power series have the same radius of convergence $R$. Finally, we obtain:

$c_{0}=f(0), c_{1}=\frac{\left({ }^{(1)} T_{\alpha} f\right)(0)}{\alpha}, \ldots, c_{n}=\frac{\left({ }^{(n)} T_{\alpha} f\right)(0)}{\alpha^{n}(n !)}$, which proves the result. 
Remark 3.5. If the power series is centered at point $t_{0}>0$, from the above theorem, it is easy to obtain:

$c_{k}=\frac{\left({ }^{k} T_{\alpha} f\right)\left(t_{0}\right)}{\alpha^{n}(k !)}$, for every $k=0,1,2, \ldots$

Finally, we will show that the conformable power series can be integrated term by term on every closed interval that contains in its interval of convergence.

Theorem 3.6. Suppose that the power series $\sum_{k=0}^{\infty} c_{k} t^{k \alpha}$ has a radius of convergence $R>0$. Then:

(i) The $\alpha$-integrated power series $\sum_{k=0}^{\infty} \frac{c_{k}}{(k+1) \alpha} t^{(k+1) \alpha}$ converges for all $t_{0} \in[0, R)$.

(ii) If $t \in[0, R)$, it is verified that

$$
\int_{0}^{t}\left(\sum_{k=0}^{\infty} c_{k} x^{k \alpha}\right) \frac{d x}{x^{1-\alpha}}=\sum_{k=0}^{\infty} \int_{0}^{t} c_{k} x^{k \alpha} \frac{d x}{x^{1-\alpha}}=\sum_{k=0}^{\infty} \frac{c_{k}}{(k+1) \alpha} t^{(k+1) \alpha}
$$

Proof.

(i) From a certain $k, \frac{t_{0}}{k+1} \leq 1$. Then, we have: $\left|\frac{c_{k}}{(k+1) \alpha} t_{0}^{(k+1) \alpha}\right| \leq \frac{t_{0}}{(k+1) \alpha}\left|c_{k} t_{0}^{k}\right|$ According to the comparison test, the integrated series converges.

(ii) Since $\sum c_{k} x^{k \alpha}$ is a series of continuous functions that converge uniformly in the closed interval $[0, t]$, we can integrate term by term. The above equality is a consequence of the convergence of $\sum \frac{c_{k}}{(k+1) \alpha} t^{(k+1) \alpha}$ at both 0 and $t$.

Example 3.2. Let $0<\alpha<1$. The power series $\sum_{K=o}^{\infty}(k+1) \alpha t^{k \alpha}$ has a radius of convergence $R=1$ since we have: $l=\lim _{k \rightarrow \infty}\left|\frac{c_{k}}{c_{k+1}}\right|=\lim _{k \rightarrow \infty} \frac{(k+1) \alpha}{(k+2) \alpha}=1$

By taking $f(t)=\sum(k+1) \alpha t^{k \alpha}$ for all $t \in[0,1)$, we have:

$$
\int_{0}^{t} f(t) \frac{d t}{t^{1-\alpha}}=\int_{0}^{t}\left(\sum_{K=0}^{\infty}(k+1) \alpha t^{k \alpha}\right) \frac{d t}{t^{1-\alpha}} \sum_{k=0}^{\infty} \int_{0}^{t}(k+1) \alpha t^{k \alpha} \frac{d t}{t^{1-\alpha}}=\sum_{k=0}^{\infty} t^{(k+1) \alpha}=\frac{t^{\alpha}}{1-t^{\alpha}}
$$

By differentiating both sides of the above equality, we obtain: $\left(T_{\alpha} f\right)(t)=$ $\left(T_{\alpha} f\right)\left(\frac{t^{\alpha}}{1-t^{\alpha}}\right)=\frac{\alpha}{\left(1-t^{\alpha}\right)^{2}}$ 


\section{Conformable Sequential Fractional Airy Differential Equation}

Consider the following conformable fractional Airy differential equation:

$$
\left.{ }^{(2)} T_{\alpha} y\right)(t)-\alpha^{2} t^{\alpha} y(t)=0
$$

where $\alpha \in[0,1)$. If $\alpha=1$, then Eq. (4.1) becomes the classical Airy differential equation, which has many applications in diffraction theory, [20]. $t=0$ is an ordinary point of (4.1). Now, by using the power series method, $[9,10]$, and proposing the following solution:

$$
y(t)=\sum_{k=0}^{\infty} c_{k} t^{k \alpha}
$$

By substituting (4.2) and its conformable fractional derivatives in (4.1), we have

$$
2 c_{2}+\sum_{k=1}^{\infty}\left[(k+2)(k+1) c_{k+2}-c_{k-1}\right] t^{k \alpha}=0
$$

The above equation implies: $c_{2}=0 ; c_{k+2}=\frac{c_{k-1}}{(k+2)(k+1)}, k=1,2,3, \ldots$

Hence, we obtain the following: $c_{2}=c_{5}=c_{8}=c_{11}=\cdots=0$

$$
\begin{gathered}
c_{3 k}=\frac{(3 k-2)(3 k-5) \ldots 1}{(3 k) !} c_{0}, k=1,2,3, \ldots \\
c_{3 k+1}=\frac{(3 k-1)(3 k-4) \ldots 1}{(3 k+1) !} c_{1}, k=1,2,3, \ldots
\end{gathered}
$$

As a result, we have the following general solution of (4.1):

$$
\begin{array}{r}
y(t)=c_{0}\left(1+\sum_{k=1}^{\infty} \frac{(3 k-2)(3 k-5) \ldots 1}{(3 k) !} t^{3 k \alpha}\right) \\
+c_{1}\left(1+\sum_{k=1}^{\infty} \frac{(3 k-1)(3 k-4) \ldots 1}{(3 k+1) !} t^{(3 k+1) \alpha}\right)
\end{array}
$$

Remark 4.1. Note that the above equation can be written in the following form:

$$
y(t)=c_{0} y_{1}(t)+c_{1} y_{2}(t)
$$

where

$$
y_{1}(t)=1+\sum_{k=1}^{\infty} \frac{(3 k-2)(3 k-5) \ldots 1}{(3 k) !} t^{3 k \alpha}, y_{2}(t)=1+\sum_{k=1}^{\infty} \frac{(3 k-1)(3 k-4) \ldots 1}{(3 k+1) !} t^{(3 k+1) \alpha}
$$


To find the radius of convergence of the series (4.3), we will use the ratio test as follows:

$$
\begin{aligned}
& \lim _{k \rightarrow \infty}\left|\frac{(3 k+3) !(3 k-2)(3 k-5) \ldots 1}{(3 k) !(3 k+1)(3 k-2) \ldots 1}\right|=\lim _{k \rightarrow \infty}(3 k+3)(3 k+2)=\infty \\
& \lim _{k \rightarrow \infty}\left|\frac{(3 k+4) !(3 k-1)(3 k-4) \ldots 1}{(3 k+1) !(3 k+2)(3 k-1) \ldots 1}\right|=\lim _{k \rightarrow \infty}(3 k+4)(3 k+3)=\infty
\end{aligned}
$$

Thus, the radius of convergence is $\infty$ in both cases.

\section{Conclusions}

The main goal of this work has been achieved by providing a generalized fractional power series formulation. The conformable fractional derivatives have been used in constructing some of important theorems and relations. The ratio test has been successfully established for this generalized formulation, and all conformable differentiability and integrability theorems of the sum of a power series have been successfully proved. All proposed theorems have been used for approximating the fractional derivatives and integrals of functions that are represented as a fractional power series. These results have been validated as efficient and simple tool for solving fractional differential equations such as the conformable fractional Airy differential equation.

\section{REFERENCES}

[1] A. Kilbas, H. Srivastava \& J. Trujillo, Theory and Applications of Fractional Differential Equations, North-Holland, New York, 2006.

[2] K.S. Miller, An Introduction to Fractional Calculus and Fractional Differential Equations, J. Wiley and Sons, New York, 1993.

[3] R. Khalil, M. Al Horani, A. Yousef \& M. Sababheh, A new definition of fractional derivative, J. Comp. Appl. Math. 264 (2014), 65-70. 
[4] T. Abdeljawad, On conformable fractional calculus. J. Comp. Appl. Math. 279 (2015), 57-66.

[5] O.S. Iyiola \& E.R. Nwaeze, Some new results on the new conformable fractional calculus with application using D’Alambert approach, Progr. Fract. Differ. Appl. 2(2) (2016), 1-7.

[6] A. Atangana, D. Baleanu \& A. Alsaedi, New properties of conformable derivative, Open Math. 13 (2015), 57-63.

[7] N. Yazici \& U. Gözütok, Multivariable Conformable fractional Calculus, Filomat 32(1) (2018), 45-53.

[8] F. Martínez, I. Martínez \& S. Paredes, Conformable Euler's Theorem on homogeneous functions. Comp. Math. Methods 1(5) (2018), 1-11.

[9] E. Ünal, A. Gökdogan \& E. Çelik, Solutions of Sequential Conformable Fractional Differential Equations around an Ordinary Point and Conformable Fractional Hermite Differential Equation, Br. J. Appl. Sci. Technol. 10(2) (2015), 1-11.

[10] M. Al Masalmeh, Series Method to solve conformable fractional Riccati Differential equations, Int. J. Appl. Math. Res. 6(1) (2017), 30-33.

[11] M. Al Horani \& R. Khalil, Total fractional differential with applications to exact fractional differential equations, Int. J. Computer Math. 95(6-7) (2018), 1444-1452.

[12] M. Al Horani, M.A. Hammad \& R. Khalil, Variations of parameters for local fractional nonhomogeneous linear-differential equations, J. Math. Computer Sci. 16 (2016), 147-153.

[13] R. Khalil, M. Al Horani \& D. Anderson, Undetermined coefficients for local differential equations, J. Math. Computer Sci. 16 (2016), 140-146.

[14] M.A. Hammad \& R. Khalil, Abel's formula and wronskian for conformable fractional differential equations, Int. J. Differ. Equ. Appl. 13(2) (2014), 177-183.

[15] M.A. Hammad \& R. Khalil, Legendre fractional differential equation and Legendre fractional polynomials, Int. J. Appl. Math. Res. 3(3) (2014), 214-219.

[16] F.S. Silva, M.D. Moreira \& M.A. Moret, Conformable Laplace Transform of Fractional Differential Equations, Axioms 7 (2018), 55. 
[17] Z. Al-Zhour, N. Al-Mutairi, F. Alrawajeh \& R. Alkhasawneh, Series solutions for the Laguerre and Lane-Emden fractional differential equations in the sense of conformable fractional derivative, Alex. Eng. J. 58(4) (2019), 1413-1420.

[18] M.A. Hammad, H. Alzaareer, H. Al-Zoubi \& H. Dutta, Fractional Gauss hypergeometric differential equation, J. Interdiscip. Math. 22(7) (2019), 1113-1121.

[19] T.M. Apostol, Calculus. Volume 1 (2nd edition), John Wiley \& Sons, 1991.

[20] W.R. Derrick \& S.I. Grossman, Elementary Differential Equations with Applications. AddisonWesley Publising Company, Inc., de Reading, 1981. 